\title{
A HOMOTOPY CLASSIFICATION OF SYMPLECTIC IMMERSIONS
}

\author{
MAHUYA DATTA \\ International Centre for Theoretical Physics \\ P.O.Box 586, 34100 Trieste, Italy \\ E-mail: datta@ictp.trieste.it
}

1. Introduction. The main result of this paper is concerning to the $C^{0}$-dense parametric $h$-principle [1] of symplectic immersions. Let $(N, \sigma)$ be a smooth symplectic manifold and $M$ a manifold with a closed $C^{\infty}$ 2-form $\omega$ on it. A smooth map $f$ : $(M, \omega) \longrightarrow(N, \sigma)$ is called symplectic if $f$ pulls back $\sigma$ onto $\omega$. Let $\operatorname{Symp}(M, N)$ denote the space of symplectic immersions of $M$ into $N$ with $C^{\infty}$ compact-open topology and $\operatorname{Symp}_{0}(T M, T N)$ denote the space of bundle monomorphisms $F: T M \longrightarrow T N$ with $C^{0}$ compact-open topology where $F$ satisfies $F^{*} \sigma=\omega$ and the underlying (continuous) map of $F$ pulls back the cohomology class of $\sigma$, denoted by $[\sigma]$, onto the cohomology class $[\omega]$. Then the differential $d$ maps $\operatorname{Symp}(M, N)$ into $\operatorname{Symp}_{0}(T M, T N)$. The main theorem may now be stated as follows.

Theorem 1.1. If $\operatorname{dim} M<\operatorname{dim} N$ then $d: \operatorname{Symp}(M, N) \longrightarrow \operatorname{Symp}_{0}(T M, T N)$ is a weak homotopy equivalence. In particular, symplectic immersions satisfy the $C^{0}$-dense parametric h-principle in the space of continuous maps $f: M \longrightarrow N$ which pull back the cohomology class of $\sigma$ onto that of $\omega$.

It is interesting to note that when $\operatorname{dim} N=2 \operatorname{dim} M$, taking $\omega$ equal to zero we obtain the following result of Lees [2].

COROLlary 1.2. The Lagrangian immersions satisfy $C^{0}$-dense parametric h-principle.

TheOREM 1.3. Let $F: T(\mathrm{Op} A) \longrightarrow T N$ be a bundle monomorphism such that $F^{*} \sigma=$ $\omega$, where $A$ is a compact set in $M$, and let the underlying map $f$ be a symplectic immersion on a neighbourhood of a compact set $B \subset A$. If the relative cohomology class $\left[f^{*} \sigma-\omega\right]$ vanishes in $H^{2}(A, B)$ then $F$ can be homotoped to a symplectic immersion such that the homotopy remains constant in a neighbourhood of $B$.

1991 Mathematics Subject Classification: 58A30, 58D10.

The paper is in final form and no version of it will be published elsewhere. 
It should be remarked that Gromov studied in $[1, \S 3.4 .2]$ a more general problem, namely the classification of $\sigma$-regular isometric immersions for an arbitrary closed 2 -form $\sigma$. The general theorem arises from the $h$-principle of some auxiliary sheaf which comes as the solution sheaf of an infinitesimally invertible differential operator, and Gromov proved this by using sophisticated machinery, for example, the Nash-Moser Implicit Function Theorem. However, the situation becomes much simplified when we restrict ourselves to isometric immersions in a symplectic manifold.

Our proof of Theorem 1.1 is based on a comment of Gromov [1, p. 327]. The proof involves sheaf theoretic technique and Moser's Stability Theorem for symplectic forms. Gromov used this technique to prove the 'Open Extension Theorem', which gives $h$-principle for a large class of partial differential relations, namely, relations admitting of open extensions which are invariant under fibre-preserving diffeomorphisms. The main idea there was to find a class of diffeotopies that would 'sharply move a submanifold locally at hypersurfaces' $[1, \S 2.2 .3]$ and at the same time would keep the extension relation invariant under its action. In the Open Extension Theorem fibre-preserving diffeotopies serve this purpose. In this specific problem the role is played by exact diffeotopies $[1, \S 3.4 .2]$. However, the relation corresponding to symplectic immersions is non-open and the sheaf of symplectic isometric immersions is not even microflexible [see Section 2]. Hence Theorem 1.1 does not follow immediately from the Open Extension Theorem. The difficulty has been bypassed here by passing to an auxiliary sheaf which is microflexible and which has the same homotopy type as the sheaf of those symplectic isometric immersions whose graphs lie in a certain predefined subspace. On the other hand, since the relation is not open, an infinitesimal solution is not necessarily a local solution. Nevertheless, Moser's Stability Theorem [4] tells us that an infinitesimal solution is isotopic to a local solution of the differential relation.

For any undefined term we refer to [1]

2. Brief review of the sheaf theoretic results. We now briefly describe the sheaf theoretic techniques to prove parametric $h$-principle. Let $\Phi$ denote the sheaf of solutions of some $r$-th order partial differential relation $\mathcal{R} \subset J^{r}(M, N)$ defined for $C^{r}$ maps $M \longrightarrow N$, and $\Psi$ the sheaf of sections of the $r$-jet bundle $J^{r}(M, N) \longrightarrow M$ with images in $\mathcal{R}$. The natural topologies on $\Phi(U)$ and $\Psi(U)$ are respectively the $C^{r}$ and $C^{0}$ compact open topologies.

Definition 2.1. The solution sheaf $\Phi$ and the relation $\mathcal{R}$ are said to satisfy parametric $h$-principle if the $r$-jet map $\jmath^{r}: \Phi \longrightarrow \Psi$ is a weak homotopy equivalence.

Before proceeding further we state some general definitions and results on topological sheaves.

Definition 2.2. Let $\mathcal{F}$ be a topological sheaf over $M$ and $A$ be a compact set in $M$. The symbol $\mathcal{F}(A)$ will denote the space of maps which are defined over some neighbourhood of $A$ in $M$; in fact it is the direct limit of the spaces $\mathcal{F}(U)$ where $U$ runs over all the open sets containing $A$. A map $f: P \longrightarrow \mathcal{F}(A)$ on a polyhedron $P$ is called continuous if there exists an open set $U \supset A$ such that each $f_{p}$ is defined over $U$ and the resulting 
map $P \longrightarrow \mathcal{F}(U)$ is continuous with respect to the given topology on $\mathcal{F}(U)$.

Definition 2.3. A sheaf $\mathcal{F}$ is called flexible if the restriction maps $\mathcal{F}(A) \longrightarrow \mathcal{F}(B)$ are Serre fibrations for every pair of compact sets $(A, B), A \supset B$. The restriction map $\mathcal{F}(A) \longrightarrow \mathcal{F}(B)$ is called a microfibration if given a continuous map $f_{0}^{\prime}: P \longrightarrow \mathcal{F}(A)$ on a polyhedron $P$ and a homotopy $f_{t}, 0 \leq t \leq 1$, of $\left.f_{0}^{\prime}\right|_{B}$ there exists an $\varepsilon>0$ and a homotopy $f_{t}^{\prime}$ of $f_{0}^{\prime}$ such that $f_{t}^{\prime} \mid \operatorname{Op} B=f_{t}$ for $0 \leq t \leq \varepsilon$. If for any pair of compact sets the restriction morphism is a microfibration then the sheaf $\mathcal{F}$ is called microflexible.

Theorem 2.4 (Sheaf Homomorphism Theorem [1, p. 77]). Let $\mathcal{F}$ and $\mathcal{G}$ be two topological sheaves defined on a manifold $M$ and let $f: \mathcal{F} \longrightarrow \mathcal{G}$ be a morphism. If both sheaves are flexible and if $f$ is a local weak homotopy equivalence then $f$ is a weak homotopy equivalence.

So to prove parametric $h$-principle for a relation $\mathcal{R}$ it suffices to show that the sheaves $\Phi$ and $\Psi$ (as defined above) are flexible and the $r$-jet map $j^{r}: \Phi \longrightarrow \Psi$ is a local weak homotopy equivalence. For any partial differential relation $\mathcal{R}$ the sheaf $\Psi$ is always flexible $[1$, p. 40]. But to prove flexibility of $\Phi$ we need to impose certain extensibility conditions on $\mathcal{R}$.

Let $M$ be embedded in a higher dimensional manifold $M^{\prime}$ and let $\mathcal{R}^{\prime}$ be a relation on $M^{\prime}$. We denote the corresponding sheaf of solutions by $\Phi^{\prime}$.

Definition 2.5. $\Phi^{\prime}$ is said to be an extension of $\Phi$ if the inclusion of $M$ in $M^{\prime}$ induces a restriction homomorphism $\alpha:\left.\Phi^{\prime}\right|_{M} \longrightarrow \Phi$; moreover, $\alpha(x)$ is a surjection for each $x \in M$.

This means that if we restrict a solution of $\mathcal{R}^{\prime}$ to $M$ we obtain a solution of $\mathcal{R}$ and moreover every local solution of $\mathcal{R}$ can be lifted to a local solution of $\mathcal{R}^{\prime}$.

Now, for a pair of compact subsets $(A, B)$ in $M$ we define the space $\Gamma(A, B)$ of compatible pairs of solutions inside $\Phi^{\prime}(B) \times \Phi(A)$. This set consists of all pairs $\left(f^{\prime}, f\right)$ such that $\alpha\left(f^{\prime}\right)=f \mid \mathrm{Op} B$.

Definition 2.6. The extension $\Phi^{\prime}$ will be called a microextension if the obvious map $\gamma: \Phi^{\prime}(A) \longrightarrow \Gamma(A, B)$ is a microfibration.

Now we explain the concept of diffeotopy sharply moving $M$ in $M^{\prime}$. It is worth recalling that the idea contained in this definition is a key point in the Smale-Hirsch Immersion Theorem.

Definition 2.7. We fix a metric $d$ on $M$. An open set in $M$ will be called small if it is contained in a ball of small radius. A class of diffeotopy $\mathcal{D}$ on $M^{\prime}$ is said to sharply move $M$ in $M^{\prime}$ if given any hypersurface $S$ lying in a small open set of $M$ and given any two positive numbers $r$ and $\varepsilon$ we can obtain a diffeotopy $\left\{\delta_{t}\right\}$ in $\mathcal{D}$ which satisfies the following conditions:

(a) $\delta_{0}$ is the identity map,

(b) each $\delta_{t}$ is identity outside an $\varepsilon$-neighbourhood of $S$,

(c) $d\left(\delta_{1}(S), M\right)>r$. 
Gromov gives the following sufficient condition for flexibility of $\Phi$ in his Main Lemma [1, p. 82] and Microextension Theorem [1, p. 85].

THEOREM 2.8. If $\Phi$ admits of a microextension $\Phi^{\prime}$ which is microflexible and if there exists a class of acting diffeotopy on $\Phi^{\prime}$ which sharply moves $M$ in $M^{\prime}$ then $\Phi$ is a flexible sheaf.

3. Defining an extension. Let $(M, \omega)$ and $(N, \sigma)$ be as in Section 1 . Then the symplectic immersions $(M, \omega) \longrightarrow(N, \sigma)$ correspond to the partial differential relation $\mathcal{R} \subset J^{(1)}(M, N)$ consisting of all 1-jets $\jmath_{x}^{1} f, x \in M$, of local immersions $f$ such that $f^{*} \sigma=\omega$ at $x$. Let $\Psi$ denote the sheaf of bundle maps $F: T M \longrightarrow T N$ which pull back the form $\sigma$ onto $\omega$. This may be identified with the sheaf of sections of $\mathcal{R}$. To obtain an extension of $\mathcal{R}$, we will first embed $(M, \omega)$ isometrically into a symplectic manifold $\left(M^{\prime}, \omega^{\prime}\right)$. We start with an $F: T M \longrightarrow T N$ in the sheaf $\Psi(M)$. Let $f: M \longrightarrow N$ be the underlying continuous map. We consider the bundle $f^{*} T N / T M$ over $M$. Observe that the total space of the bundle, say $X$, has the same dimension as $N$. Now we can construct a symplectic form $\omega^{\prime}$ on it. We first extend the bundle map $F$ to a bundle morphism $F^{\prime}: T X \mid M \longrightarrow T N$ such that $F^{\prime}$ maps fibres of $T X \mid M$ isomorphically onto the fibres of $T N$. The form ${F^{\prime *}}^{*}$ restricts to $\omega$ on $M$ and hence can be extended to a closed form $\omega^{\prime}$ on some neighbourhood $M^{\prime}$ of $M$ in $X . M^{\prime}$ may be taken to be a tubular neighbourhood of $M$ in $X$ so that the inclusion $i: M \longrightarrow M^{\prime}$ is a homotopy equivalence. Since ${F^{\prime *}}^{*}$ is non-degenerate so is $\omega^{\prime}[5]$. So $(M, \omega)$ is isometrically embedded in the symplectic manifold $\left(M^{\prime}, \omega^{\prime}\right)$.

We denote the sheaf of symplectic isometric immersions of $(M, \omega)$ in $(N, \sigma)$ by $\mathcal{S}$ and that of $\left(M^{\prime}, \omega^{\prime}\right)$ in $(N, \sigma)$ by $\mathcal{S}^{\prime}$. Let $\mathcal{R}^{\prime}$ denote the space of 1 -jets of germs of symplectic immersions of $\left(M^{\prime}, \omega^{\prime}\right)$ in $(N, \sigma)$ and $\Psi^{\prime}$ the sheaf of section of $\mathcal{R}^{\prime}$.

Proposition 3.1. $\mathcal{S}^{\prime}$ is an extension of $\mathcal{S}$.

Proof. It is easy to see that the isometric embedding of $(M, \omega)$ in $\left(M^{\prime}, \omega^{\prime}\right)$ induces a morphism $\alpha:\left.\mathcal{S}^{\prime}\right|_{M} \longrightarrow \mathcal{S}$. To prove that $\alpha(x): \mathcal{S}^{\prime}(x) \longrightarrow \mathcal{S}(x)$ is onto we start with a local symplectic immersion $f$ at a point $x \in M$. Let $\bar{f}$ be any extension of $f$ to a local immersion in $M^{\prime}$. Then, since dimension of $M^{\prime}$ is the same as the dimension of $N$, the form $\bar{\omega}=\bar{f}^{*} \sigma$ is a symplectic form. Now the two linear symplectic forms $\bar{\omega}_{x}$ and $\omega_{x}^{\prime}$ defined on $T_{x} M^{\prime}$ coincide on the subspace $T_{x} M$. Hence there exists a linear isomorphism $l$ of $T_{x} M^{\prime}$ which pulls back $\bar{\omega}_{x}$ onto $\omega_{x}^{\prime}$ and keeps $T_{x} M$ pointwise fixed. We consider the germ of a local map $f^{\prime}$ whose 1 -jet at $x$ equals to $\jmath_{x}^{1} \bar{f} \circ l$ so that $\jmath_{x}^{1} f^{\prime} \in \mathcal{R}^{\prime}$. By construction the jet $\jmath_{x}^{1} f^{\prime}$ projects onto $\jmath_{x}^{1} f \in \mathcal{R}$. Moreover we may assume without loss of generality that $f^{\prime}$ extends $f$. So we have the following:

- $f^{\prime *} \sigma=\omega^{\prime}$ at $x$.

- $f^{\prime}$ equals $f$ on $U \cap M$, where $U$ is the domain of $f$. Hence, pullbacks of both the forms $f^{\prime *} \sigma$ and $\omega^{\prime}$ to $M$ are the same.

Therefore, by the Relative Poincaré Lemma, we obtain a 1-form $\varphi$ on a neighbourhood, say $\widetilde{U}$, of $x$ in $U$ such that $d \varphi={f^{\prime *}}^{*}-\omega^{\prime}$ and $\varphi \mid \widetilde{U} \cap M=0$. Now, by applying Moser's Theorem [4] we get a diffeomorphism $\delta$ on a neighbourhood, say $U^{\prime}$, of $x$ in $\widetilde{U}$, such that 
$\delta^{*}\left(f^{\prime *} \sigma\right)=\omega^{\prime}, \delta \mid U^{\prime} \cap M$ is identity, and $d \delta_{x}=$ id. Then $f^{\prime} \circ \delta$ is the required extension of $f$.

Proposition 3.2. The 1-jet map $j^{1}: \mathcal{S} \longrightarrow \Psi$ is a local weak homotopy equivalence.

Proof. The main ingredient of the proof is Moser's Theorem on the stability of symplectic forms in a cohomology class. Consider the map $\rho=e \circ \jmath^{1}: \Phi(x) \longrightarrow \Psi(x) \longrightarrow \mathcal{R}_{x}$, where the space $\mathcal{R}_{x}$ consists of 1 -jets of symplectic immersions at $x$, and $e$ is the evaluation map at $x$. We shall prove that $\rho$ induces an injective map between homotopy groups. It will then imply that the induced map $J_{*}^{1}$ on homotopy groups is also injective. Injectiveness of $\rho_{*}$ may be proved proceeding as in du Plessis [3] and using the following observation. Let $\varphi_{p}: M \longrightarrow N, p \in P$, be a continuous family of smooth maps parametrized by a polyhedron $P$ such that $\varphi_{p}^{*} \sigma=\omega$ at $x$. By the above lemma we can extend $\varphi_{p}$ to a neighbourhood of $x$ in $M^{\prime}$ as $\varphi_{p}^{\prime}$ such that $\varphi_{p}^{\prime *} \sigma=\omega^{\prime}$ at $x$. We set $\omega_{p}^{\prime}=\varphi_{p}^{\prime *} \sigma$. Then $\omega_{p}^{\prime}=\omega^{\prime}$ at $x$ for each $p \in P$. Now by Moser's Theorem we get a family of diffeomorphisms $\delta_{p}$ (homotopic to the identity), defined on a neighbourhood of $x$ such that $\delta_{p}^{*} \omega_{p}^{\prime}=\omega^{\prime}, \delta_{p}(x)=x$ and $d \delta_{p} \mid T_{x} M^{\prime}=$ id. Define $\bar{\varphi}_{p}=\left.\varphi_{p}^{\prime} \circ \delta_{p}\right|_{M}$ on Op $x$. Then $\bar{\varphi}_{p}$ 's are isometric immersions on $\operatorname{Op} x$ and $\jmath_{x}^{1} \varphi_{p}=\jmath_{x}^{1} \bar{\varphi}_{p}$. Moreover, if some $\varphi_{p}$ is isometric on a neighbourhood of $x$, we may get $\bar{\varphi}_{p}=\varphi_{p}$ on $\mathrm{Op} x$ in $M$.

We now prove that $j_{*}^{1}$ is surjective. Let $\Gamma$ denote the sheaf of smooth maps from $M$ to $N$. This is a sheaf over $M$. Consider the parametric sheaf $\Gamma^{M}$ over $M \times M$ which is defined as follows: For open subsets $U, V \subset M$ we set $\Gamma^{M}(U \times V)$ equal to $\Gamma(U)^{V}$, which is the space of continuous maps $V \longrightarrow \Gamma(U)$. Now take the restriction of $\Gamma^{M}$ to the diagonal. We shall denote this sheaf by $\Gamma^{*}$, and call it the associated sheaf of $\Gamma$. Observe that $\Gamma^{*}(x)$ is the direct limit of the spaces $\Gamma(U)^{U}$ where $U$ runs over open neighbourhoods of $x$ in $M$. Thus a local section in $\Gamma^{*}$ can be conceived as a continuous family of germs $\varphi_{x} \in \Gamma(U), x \in U$. It can be proved that the canonical inclusion of $\Gamma$ in $\Gamma^{*}$, given by $\varphi \mapsto\{u \mapsto \varphi\}$, is a weak homotopy equivalence (see [1, p. 76]). (The above construction is equally true for an arbitrary sheaf.) Returning to the proof of surjectiveness of $j_{*}^{1}$, we split $j^{1}$ in the following way:

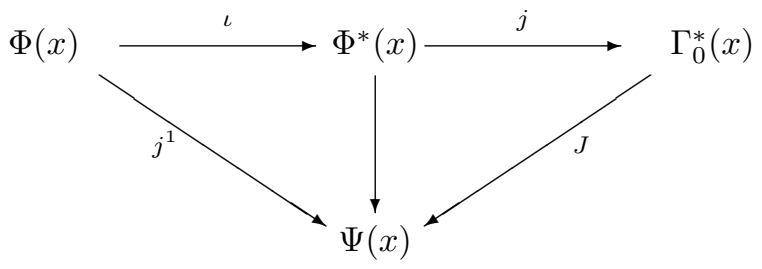

where $\Phi^{*}$ is the associated sheaf of $\Phi$ and $\Gamma_{0}^{*}(x)$ is the subspace of $\Gamma^{*}(x)$ consisting of all those families of germs $\left\{\varphi_{u}: u \in \mathrm{Op} x\right\}$ for which $\varphi_{u}$ is a local immersion and $\varphi_{u}^{*} \sigma=\omega$ at $u$, in other words $\jmath_{u}^{1} \varphi_{u} \in \mathcal{R}$. Thus it is easy to see that any section in $\Psi(x)$ gives rise to a section in $\Gamma_{0}^{*}(x)$. Hence $J$ is onto. (The same technique can be applied to show that $J_{*}$ is onto at each homotopy level.) Now we shall show that the map $j$ induces surjective homomorphism in the homotopy, which will complete our proof. To prove this, it is enough to consider the zeroeth homotopy level. To this end, take a family $\left\{\varphi_{u}: u \in U\right\}$ as above where $U$ is an open neighbourhood of $x$. We may suppose without 
loss of generality that each $\varphi_{u}$ is defined on the same open subset $U$. Now, using Moser's Theorem we can deform the family $\left\{\varphi_{u}\right\}$ to a family $\left\{\bar{\varphi}_{u}\right\}$ of symplectic immersions in $\Phi(\widetilde{U})$ such that $\jmath_{u}^{1} \bar{\varphi}_{u}=\jmath_{u}^{1} \varphi_{u}$ for all $u \in \widetilde{U}$, where $\widetilde{U}$ is an open neighbourhood of $x$ in $U$. Moreover, for each $u$, the homotopy between $\jmath^{1} \bar{\varphi}_{u}$ and $\jmath^{1} \varphi_{u}$ is constant at $u$. The family $\left\{\bar{\varphi}_{u}\right\}$ defines a section in $\Phi^{*}(x)$. So we have proved that every path component of $\Gamma_{0}^{*}(x)$ intersects $\Phi^{*}(x)$. Thus $j_{*}$ is onto at the zeroeth homotopy level.

However, the extension sheaf $\mathcal{S}^{\prime}$ is not microflexible, as it can be seen from the following example.

Example 3.3. Consider the standard embedding of the closed unit disc in $\mathbf{R}^{2}$. If we deform it near the boundary by pushing it inside then it (the homotopy) cannot be extended symplectically on the whole of the disc.

This phenomenon may be explained as follows: If $f_{0}$ is a symplectic immersion over Op $A$ and $f_{t}$ a homotopy of $f_{0}$ such that $f_{t} \mid \mathrm{Op} B$ is a symplectic immersion, then the relative cohomology class of $f_{t}^{*} \sigma-\omega$ in $H^{2}(A, B)$ determines the obstruction to extending $f_{t} \mid \mathrm{Op} B$ to Op $A$ as a symplectic immersions. If the cohomology class $\left[f_{t}^{*} \sigma-\omega\right]=0 \in$ $H^{2}(A, B)$ then there exists a smooth of 1-forms $\alpha_{t}$ such that $\alpha_{t}$ vanishes on $\operatorname{Op} B$ and $f_{t}^{*} \sigma-\omega=d \alpha_{t}$. Then Moser's Stability Theorem applies and we can lift $f_{t} \mid \operatorname{Op} B$ over $A$ as symplectic immersion.

Since $\mathcal{S}^{\prime}$ is not microflexible we cannot apply the sheaf theoretic techniques (described in Section 2) on it. However, we shall see in the following section that there exists a topological sheaf on $M^{\prime}$ naturally associated to a subspace of the space of symplectic immersions which do satisfy microflexibility and has the same homotopy type as $\mathcal{S}^{\prime}$.

4. Construction of the Auxiliary Sheaf. Since both the differential 2-forms $\sigma$ and $\omega^{\prime}$ are symplectic, the product form $p_{2}^{*} \sigma-p_{1}^{*} \omega^{\prime}$ on $M^{\prime} \times N$ is a symplectic form, where $p_{1}$ and $p_{2}$ are respectively the projection maps of $M^{\prime} \times N$ onto the first and the second factor. We shall denote this product symplectic form by $\sigma-\omega^{\prime}$. If $f: M \longrightarrow N$ is a symplectic isometric immersion then its graph map $g=(1, f): M^{\prime} \longrightarrow M^{\prime} \times N$ is a Lagrangian section of $\left(M^{\prime} \times N, \sigma-\omega^{\prime}\right)$, and this correspondence is bijective.

In the rest of this section we assume that the symplectic form $\sigma-\omega^{\prime}$ is exact (which is equivalent to saying that $\sigma$ and $\omega$ are exact). Let $\tau$ be a 1 -form such that $\sigma-\omega^{\prime}=d \tau$. We construct the sheaf of exact Lagrangian sections as follows: This consists of pairs $(g, \varphi)$, where $g: M^{\prime} \longrightarrow M^{\prime} \times N$ is a section such that the underlying map $f=p_{2} \circ g: M^{\prime} \longrightarrow N$ is an immersion, and $\varphi$ is a function on $M^{\prime}$ such that $g^{*} \tau=d \varphi$. We denote the sheaf of such pairs by $\mathcal{E}^{\prime}$ and call it the sheaf of $\tau$-exact Lagrangian sections. Observe that $\mathcal{S}^{\prime}$ and $\mathcal{E}^{\prime}$ are locally homotopically equivalent since the germ of a Lagrangian section at a point denotes a germ of an exact Lagrangian section; moreover the space of primitives $\varphi$ for a $\tau$-exact Lagrangian section $g$ (meaning that $\varphi$ satisfies the relation $g^{*} \tau=\varphi$ ) is isomorphic to $\mathbf{R}$. Consequently, the sheaf of sections corresponding to the relation, of which $\mathcal{E}^{\prime}$ is the solution sheaf, has the same homotopy type as $\Psi^{\prime}$. We now prove

Proposition 4.1. The sheaf $\mathcal{E}^{\prime}$ of $\tau$-exact Lagrangian sections is microflexible.

Pro of. Let $(A, B)$ be a pair of compact sets in $M^{\prime}$. Let $g^{\prime}$ be a $\tau$-exact Lagrangian 
section over a $A$ (meaning that it is defined on a neighbourhood of $A$ ) such that $g^{\prime *} \tau=d \varphi^{\prime}$ for a 0 -form $\varphi^{\prime}$, and $\left(g_{t}, \varphi_{t}\right)$ a homotopy of $\left(g^{\prime}, \varphi^{\prime}\right) \mid$ Op $B$ in $\mathcal{E}^{\prime}$.

We first prove the following simple lemma.

LEMMA 4.2. Let $g_{t}$ be a homotopy of $\tau$-exact Lagrangian sections. If $g_{0}$ is $\tau^{\prime}$-exact Lagrangian for a 1-form $\tau^{\prime}$ satisfying $\sigma-\omega^{\prime}=d \tau^{\prime}$, then $g_{t}$ is also $\tau^{\prime}$-exact Lagrangian for each $t$.

Pr o of. Two such forms $\tau$ and $\tau^{\prime}$ differ by a closed 1 -form $c$ on $M^{\prime} \times N$. So, we have the following relation

$$
g_{t}^{*} \tau^{\prime}=g_{t}^{*} \tau+g_{t}^{*} c
$$

for every $t$. Then, by hypothesis, $g_{0}^{*} c$ is an exact form. Since $c$ is closed, $g_{t}^{*} c$ is also exact. Consequently $g_{t}^{*} \tau^{\prime}$ is exact for each $t$.

Proof of Proposition 4.1 (continued). Now, by the standard theory of Lagrangian submanifolds [4], there exists a neighbourhood $W$ of the Lagrangian submanifold $L^{\prime}=\operatorname{Im} g^{\prime}$ such that $(W, d \tau)$ is symplectomorphic to a neighbourhood of the zero section $Z_{L^{\prime}}$ in the cotangent bundle $\left(T^{*} L^{\prime}, d \theta_{L^{\prime}}\right)$ with the standard symplectic form $d \theta_{L^{\prime}}$ on it. Under this correspondence, the Lagrangian submanifolds in $W$ are mapped onto the closed forms (near $Z_{L^{\prime}}$ ), whereas the $\tau^{\prime}=\delta^{*} \theta_{L^{\prime}}$-exact Lagrangians correspond to exact forms on $L^{\prime}$. Clearly the sheaf of exact 1-forms is microflexible. Hence we can obtain lifts $g_{t}^{\prime}$ of $g_{t}$ (for $t$ small enough) which are $\tau^{\prime}$-exact Lagrangian sections. By the Lemma above they are also $\tau$-exact. Moreover, for small $t$, the underlying maps will be immersions on Op $A$. Now, we can choose a homotopy $\varphi_{t}^{\prime}$ on Op $A$ such that $g_{t}^{\prime *} \tau=d \varphi_{t}^{\prime}$. On Op $B$, we have $d \varphi_{t}^{\prime}=d \varphi_{t}$. Hence $\varphi_{t}^{\prime}-\varphi_{t}=c_{t}$, where $c_{t}$ is a closed 0 -form, that is a constant. So we may replace $\varphi_{t}^{\prime}$ by $\varphi_{t}^{\prime}-c_{t}$. The homotopy $\left(g_{t}^{\prime}, \varphi_{t}^{\prime}-c_{t}\right)$ is the required lift.

We shall now describe a class of diffeotopy which would act on the sheaf $\mathcal{E}^{\prime}$ and at the same time sharply move a submanifold of $M^{\prime}$ of positive codimension. Since $\omega^{\prime}$ is symplectic we have an isomorphism $I_{\omega^{\prime}}: \mathcal{X}\left(M^{\prime}\right) \longrightarrow \Lambda^{1}\left(M^{\prime}\right)$ from the space of vector fields $\mathcal{X}\left(M^{\prime}\right)$ onto the space of 1 -forms $\Lambda^{1}\left(M^{\prime}\right)$. A $C^{\infty}$ diffeotopy $\delta_{t}$ of $M^{\prime}$ is called exact if $\delta_{0}$ is identity and if $\delta_{t}^{\prime}=\frac{d \delta_{t}}{d t}$ is a Hamiltonian vector field for each $t$. So we can write $\delta_{t}^{\prime} \cdot \omega^{\prime}\left(=I_{\omega^{\prime}}\left(\delta_{t}^{\prime}\right)\right)=d \alpha_{t}$ for some smooth family of exact 1-form $d \alpha_{t}$ on $M^{\prime}$. If $\alpha_{t}$ can be chosen to be identically zero on the open subset where $\delta_{t}$ is constant then such a diffeotopy is called a strictly exact diffeotopy.

Proposition 4.3. The strictly exact diffeotopies of $M^{\prime}$ act on the sheaf $\mathcal{E}^{\prime}$.

P r o o f. Let $\delta_{t}$ be a strictly exact diffeotopy on $M^{\prime}$. We define a diffeotopy $\bar{\delta}_{t}$ on $M^{\prime} \times N$ by $\bar{\delta}_{t}(x, y)=\left(\delta_{t}(x), y\right)$, where $x \in M^{\prime}$ and $y \in N$. It follows that $\bar{\delta}_{t}^{\prime} \cdot\left(\sigma-\omega^{\prime}\right)$ is exact for each $t$. Let $\alpha_{t}$ be a smooth family of 0 -forms on $M^{\prime} \times N$ satisfying $\bar{\delta}_{t}^{\prime} \cdot\left(\sigma-\omega^{\prime}\right)=d \alpha_{t}$. Then,

$$
\begin{aligned}
\frac{d}{d t}\left(\bar{\delta}_{t}^{*} \tau\right)=\mathcal{L}_{\bar{\delta}_{t}^{\prime}} \tau & =d\left(\bar{\delta}_{t}^{\prime} \cdot \tau\right)+\bar{\delta}_{t}^{\prime} \cdot d \tau=d\left(\bar{\delta}_{t}^{\prime} \cdot \tau\right)+\bar{\delta}_{t}^{\prime} \cdot \sigma-\bar{\delta}_{t}^{\prime} \cdot \omega^{\prime} \\
& =d\left(\bar{\delta}_{t}^{\prime} \cdot \tau\right)+d \alpha_{t}=d\left(\bar{\delta}_{t}^{\prime} \cdot \tau+\alpha_{t}\right) .
\end{aligned}
$$

If we define $\varphi_{t}=\int_{0}^{t}\left(\bar{\delta}_{t}^{\prime} \cdot \tau+\alpha_{t}\right) d t$ then $\bar{\delta}_{t}^{*} \tau=\tau+d \varphi_{t}$. Now we are in a position to define 
the action. For $(g, \varphi) \in \mathcal{E}^{\prime}$ and $\delta_{t}$ as above, we set

$$
\delta_{t}^{*}(g, \varphi)=\left(\delta_{t}^{*} g,\left(\delta_{t}^{-1}\right)^{*}\left(\varphi+g^{*} \varphi_{t}\right)\right)
$$

where $\delta_{t}^{*} g=\bar{\delta}_{t} \circ g \circ \delta_{t}^{-1}$.

Proposition 4.4. The exact diffeotopies of the symplectic manifold $\left(M^{\prime}, \omega^{\prime}\right)$ sharply move $M$ in $M^{\prime}$.

P r o of. (Gromov) To move a closed hypersurface $S$ lying in a small open set $U$ of $M$ we start with a vector $\partial_{0} \in T_{x_{0}}\left(M^{\prime}\right)$ transversal to $U$ in $M^{\prime}$. This $\partial_{0}$ extends to an exact field $\partial=I_{\omega^{\prime}}^{-1}(d H)$ on which is transversal to $U$, since $U$ is chosen small. In order to make the corresponding exact isotopy $\delta_{t}$ sharply moves $S$, we take the union $S_{\varepsilon}=\cup_{t} \delta_{t}(S) \in M^{\prime}$ over $t \in[0, \varepsilon]$ and then multiply the Hamiltonian $H$ by a properly chosen $C^{\infty}$ function $a$ on $M^{\prime}$ which vanishes outside an arbitrarily small neighbourhood of Op $S_{\varepsilon}$ and which equals one in a smaller neighbourhood of $S_{\varepsilon}$. This makes the diffeotopy corresponding to the field $I_{\omega^{\prime}}(d(a H))$ as sharp as we want.

Now applying the Main Lemma of Gromov [1, p. 82] we may conclude from above that

Proposition 4.5. The sheaf $\left.\mathcal{E}^{\prime}\right|_{M}$ is flexible.

It then follows from the Sheaf Homomorphism Theorem that $\left.\mathcal{E}^{\prime}\right|_{M}$ satisfies parametric $h$-principle.

Let $\mathcal{E}$ be the sheaf of pairs $(g, \varphi)$ on $M$, where $g: M \longrightarrow M^{\prime} \times N$ is a section such that its underlying map is an immersions and $\varphi$ is a function on $M$ satisfying the relation $g^{*} \tau=d \varphi$. To descend $h$-principle from $\left.\mathcal{E}^{\prime}\right|_{M}$ to $\mathcal{E}$ we observe that

Proposition 4.6. $\mathcal{E}^{\prime}$ is a microextension of $\mathcal{E}$.

P r o of. From Proposition 3.1 and the discussion preceeding Proposition 4.1 it follows that $\mathcal{E}^{\prime}$ is an extension of $\mathcal{E}$. To prove that $\mathcal{E}^{\prime}$ is a microextension of $\mathcal{E}$ we consider a lifting problem

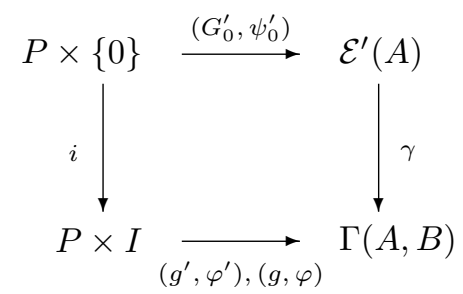

where $\alpha \circ\left(G_{0}^{\prime}, \psi_{0}^{\prime}\right)=\left(g_{0}, \varphi_{0}\right)$ and $\left(h_{0}^{\prime}, \psi_{0}^{\prime}\right) \mid \mathrm{Op} B=\left(g_{0}^{\prime}, \varphi_{0}^{\prime}\right)$ and where $\Gamma(A, B)$ is a subset of $\mathcal{E}^{\prime}(B) \times \mathcal{E}(A)$ consisting of compatible solutions as defined in Section 2. (To avoid too many symbols we assume $P$ to be a point and denote $g(t)$ by $g_{t}$ and so on.) We shall denote the underlying maps of $G_{0}^{\prime}, g_{t}^{\prime}$ and $g_{t}$ by $F_{0}^{\prime}, f_{t}^{\prime}$ and $f_{t}$. Since they are immersions (which correspond to an open differential relation), we can obtain a lift of the corresponding microextension problem for immersions. Let us denote the lift by $F_{t}$, where $0 \leq t \leq \varepsilon$ for some positive number $\varepsilon \leq 1$. Now each $F_{t}$ being immersion between equidimensional spaces, pulls back $\sigma$ onto a symplectic form on a neighbourhood of $A$. Let us set $F_{t}^{*} \sigma=\omega_{t}^{\prime}$. We denote the corresponding graph map by $G_{t}$. Then we have the 
relation $F_{t}^{*} \sigma-\omega^{\prime}=d G_{t}^{*} \tau$. On the other hand we obtain a homotopy $\psi_{t}^{\prime}$ of $\psi_{0}^{\prime}$ such that $\psi_{t}^{\prime}$ coincides with $\varphi_{t}$ and $\varphi_{t}^{\prime}$ on the relevant spaces. The 1 -form $\alpha_{t}$ defined by $\alpha_{t}=G_{t}^{*} \tau-d \psi_{t}^{\prime}$ satisfies the following

(a) $\alpha_{0}=0$

(b) $\alpha_{t}$ vanishes on some open neighbourhood of $A$ in $M$,

(c) $\alpha_{t}$ vanishes on an open neighbourhood of $B$ in $M^{\prime}$

(d) $F_{t}^{*} \sigma-\omega^{\prime}=d \alpha_{t}$.

Consider the vector fields $X_{t}=I_{\omega_{t}}^{-1}\left(\frac{d \alpha_{t}}{d t}\right)$. The vector field vanishes on $\mathrm{Op}_{M} A$ as well as on $\mathrm{Op}_{M^{\prime}} B$. Hence it can be integrated on a neighbourhood of $A$ in $M^{\prime}$ to obtain a family of diffeomorphisms $\left\{\delta_{t} ; 0 \leq t \leq \widetilde{\varepsilon}\right\}$ for some $\widetilde{\varepsilon} \leq \varepsilon$ such that

(e) $\delta_{0}$ is identity on $\mathrm{Op}_{M^{\prime}} A$,

(f) $\delta_{t} \mid \mathrm{Op}_{M} A=\mathrm{id}$

(g) $\delta_{t} \mid \mathrm{Op}_{M^{\prime}} B=\mathrm{id}$

(h) $\delta_{t}^{*} \omega_{t}^{\prime}=\omega^{\prime}$.

The required partial lift of the original lifting homotopy problem can now be given by the graph map of $F_{t}^{\prime}=F_{t} \circ \delta_{t}$. In fact, Since $F_{t}^{\prime}$ is a symplectic immersion $G_{t}^{\prime *} \tau$ is closed. On the other hand, $i: M \longrightarrow M^{\prime}$ induces an isomorphism $i^{*}: H_{d e R}^{2}\left(M^{\prime}\right) \longrightarrow H_{d e R}^{2}(M)$, and we know from our initial data that $i^{*}{G^{\prime}}_{t}^{*} \tau$ is exact. Hence, $G^{\prime *} \tau$ is also exact. It is now a trivial matter to fix $\psi_{t}^{\prime}$.

The Microextension Theorem of Gromov [1, p. 85] now implies that the sheaf $\mathcal{E}$ is flexible. We have already proved the local $h$-principle in Proposition 3.2. So again appealing to the Sheaf Homomorphism Theorem we may conclude that $\mathcal{E}$ satisfies parametric $h$-principle.

Finally we prove

Proposition 4.7. $\mathcal{E}(M)$ has the same homotopy type as the space $\mathcal{S}(M)$ of symplectic isometric immersions.

Proof. Consider the following sequence of maps between the function spaces: $\left.\left.\left.\mathcal{E}^{\prime}\right|_{M} \stackrel{\left(p_{2}\right)_{*}}{\longrightarrow} \mathcal{S}^{\prime}\right|_{M} \stackrel{J^{1}}{\longrightarrow} \Psi^{\prime}\right|_{M}$. The $C^{0}$-dense parametric $h$-principle for $\left.\mathcal{E}^{\prime}\right|_{M}$ says that the composition is a weak homotopy equivalence. Hence $\left(p_{2}\right)_{*}$ induces injective maps between homotopy groups. On the other hand, given any symplectic immersion $f$ near $M$ in $M^{\prime}$ we can obtain a $\tau$-exact Lagrangian section $\left.g \in \mathcal{E}^{\prime}\right|_{M}$ such that $p_{2} \circ g$ is arbitrarily $C^{0}$-close to $f$. In particular we may choose $g$ within the neighbourhood of graph $f$ which is symplectomorphic to the neighbourhood of the zero section $Z_{M^{\prime}}$ in $T^{*}\left(M^{\prime}\right)$ (see Proposition 4.1). Hence $p_{2} \circ g$ can be homotoped within the space $\mathcal{S}^{\prime}$ to $f$. In fact, $g$ corresponds to a closed form whereas graph $f$ corresponds to the zero section. We denote the corresponding forms by the same sympbols. The homotopy $(1-t) g$ brings $g$ onto graph $f$ within the space of Lagrangian sections as multiplication by $t$ takes closed forms to closed forms, which correspond to Lagrangian sections of $M^{\prime} \times N \longrightarrow M^{\prime}$ provided they are sufficiently $C^{\infty}$ close to the zero form. This observation proves that $\left(p_{2}\right)_{*}$ induces an isomorphism between the homotopy groups.

Proceeding as in Proposition 4.6 we may observe that both the restriction maps 
$\mathcal{S}^{\prime}(M) \longrightarrow \mathcal{S}(M)$ and $\mathcal{E}^{\prime}(M) \longrightarrow \mathcal{E}(M)$ are fibration. Moreover, for any $g \in \mathcal{E}$, the fibres in $\mathcal{E}^{\prime}(M)$ and $\mathcal{S}^{\prime}(M)$ over $g$ and $p_{2} \circ g$ respectively are homotopically equivalent. We have proved above that $\mathcal{E}^{\prime}(M)$ and $\mathcal{S}^{\prime}(M)$ are of the same weak homotopy type. Hence using homotopy exact sequence of fibrations we conclude that $\mathcal{E}(M)$ and $\mathcal{S}(M)$ are also of the same weak homotopy type.

This leads us to the following intermediate theorem.

THEOREM 4.8. If the differential forms $\sigma$ and $\omega$ are exact then the space of symplectic immersions of $M$ into $N$ satisfies parametric h-principle.

5. Proof of the main theorem. Let us now go back to our case where $\sigma-\omega$ is not necessarily exact on $M \times N$. However, if $f: M \longrightarrow N$ is a continuous map such that $f^{*}[\sigma]=[\omega]$ then $f$ can be extended to a map $f^{\prime}: M^{\prime} \longrightarrow N$ such that $f^{\prime *}[\sigma]=\left[\omega^{\prime}\right]$. Then in a neighbourhood, say $W$, of graph $f$ there exists a 1 -form $\tau$ such that $\sigma-\omega^{\prime}=d \tau$. We shall denote the sheaf of symplectic immersions $M \times N$ whose graphs lie in $W$ by the symbol $\mathcal{S}_{W}$. Then from the discussion of the previous section it follows that $\mathcal{S}_{W}$ satisfies parametric $h$-principle. We now come to the proof of Theorem 1.1.

Proof of Theorem 1.1. It remains only to prove the injectivity of the maps $d_{*}: \pi_{i}(\mathcal{S}(M)) \longrightarrow \pi_{i}\left(\operatorname{Symp}_{0}(T M, T N)\right)$ for each integer $i$. Let $f_{0}$ and $f_{1}$ be two symplectic immersions on $M$ such that their differentials $d f_{0}$ and $d f_{1}$ are homotopic in $\operatorname{Symp}_{0}(T M, T N)$; that is, there exists a homotopy $F_{t}: T M \longrightarrow T N$ such that $F_{t}^{*} \sigma=\omega$ for each $t$ and the underlying maps $f_{t}: M \longrightarrow N$ satisfies $f_{t}^{*}[\sigma]=[\omega]$. For each $t$ we can choose a neighbourhood $W_{t}$ of graph $f_{t}$ on which $\sigma-\omega$ is exact. Then the sheaves $\mathcal{S}_{t}\left(=\mathcal{S}_{W_{t}}\right)$ satisfy the parametric $h$-principle. We can cover the set $\bigcup_{t} f_{t}(M)$ by finitely many such $W_{t}$ 's such that any two consecutive ones (ordered by the real number) intersect in a set which contains completely the graph of some $f_{t}$. Without any loss of generality we may assume that the neighbourhoods $\left\{W_{1}, W_{2}\right\}$ have this property. Let, for some $t_{0}$, the graph of $f_{t_{0}}$ lie in $W_{1} \cap W_{2}$. Then by $h$-principle for the sheaf $\mathcal{S}_{W_{1} \cap W_{2}}$ we obtain a symplectic immersion $f C^{0}$-close to $f_{t_{0}}$ such that the differentials $d f$ and $F_{t_{0}}$ are homotopic within $\operatorname{Symp}_{0}(T M, T N)$ and the underlying maps of the homotopy have their graphs in $W_{1} \cap W_{2}$. Then applying parametric $h$-principle for $\mathcal{S}_{1}$ we conclude that $f$ and $f_{0}$ are homotopic within the space $\mathcal{S}_{1}$. On the other hand $f$ is homotopic to $f_{1}$ within the space $\mathcal{S}_{2}$. Joining these two homotopies we obtain a homotopy between $f_{0}$ and $f_{1}$ in the space of symplectic immersions. This proves that the differential $d$ induces an isomorphism between the homotopy groups at the zero level.

Working with a family of such maps parametrized by spheres $S^{i}$, we can similarly prove the isomorphism between the higher homotopy groups of the relevant spaces which gives the desired $h$-principle.

We now prove the relative or extension version of $h$-principle for symplectic immersions.

Proof of Theorem 1.3. Since $\left[f^{*} \sigma-\omega\right]$ vanishes in $H^{2}(A, B)$, there is a 1 -form $\varphi$ vanishing on $\operatorname{Op} B$ such that $f^{*} \sigma-\omega=d \varphi$. Hence, for a proper choice of $W$ and $\tau$, $\sigma-\omega=d \tau$ on $W$ and $g=(1, f) \mid \operatorname{Op} B$ is in $\mathcal{E}_{W, \tau}(B)$. Now consider the following diagram 
where the horizontal arrows are weak homotopy equivalences and the vertical ones are fibrations.

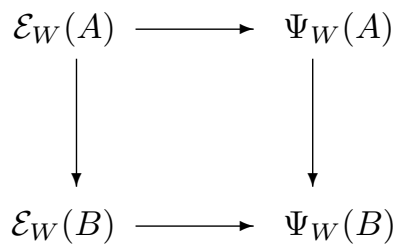

Hence the fibres over $\left.g\right|_{B}$ and $\left.d f\right|_{T B}$ are also weak homotopy equivalent. The theorem follows as $F$ lies in the fibre over $\left.d f\right|_{T B}$.

Acknowledgement. I would like to thank Professor M. Gromov for his many useful suggestions and comments.

\section{References}

[1] M. Gromov, Partial Differential Relations, Ergeb. Math. Grenzgeb. (3) 9 (1986).

[2] J. Lees, On the Classification of Lagrange Immersions, Duke Math. J. 43 (1976), 217-224.

[3] A. du Plessis, Homotopy Classification of Regular Sections, Compositio Math. 32 (1976), 301-333.

[4] A. Weinstein, Symplectic Manifolds and their Lagrangian Submanifolds, Adv. Math. 6 (1971), 329-346.

[5] A. Weinstein, Lectures on Symplectic Manifolds, North Carolina, Regional Conference Series in Math. 29, A.M.S., Providence, 1977. 